\title{
A Educação Médica entre mapas e âncoras: a aprendizagem significativa de David Ausubel, em busca da Arca Perdida*
}

\author{
The Medical education between maps and \\ anchors: David Ausubel Meaningful \\ Learning, the quest for the Lost Ark
}

PALAVRAS-CHAVE:

Aprendizagem;

Cognição;

Educação Médica.

KEYWORDS

Learning,

Cognition;

Medical Education

Recebido em: 06/08/2007

Aprovado em: 27/09/2007

$105 \frac{\text { REVISTA BRASILEIRA DE EDUCAÇÃO MÉDICA }}{32(1)+105-111 ; 2008}$

\author{
Andréia Patrícia Gomes \\ Udson Chandler Dias-Coelho ${ }^{1}$ \\ Priscila de Oliveira Cavalheiro ${ }^{1}$ \\ Cristina Angélica Nunes Gonçalvez \\ Giselle Rôças ${ }^{2}$ \\ Rodrigo Siqueira-Batista ${ }^{3}$
}

\section{RESUMO}

O processo de aprendizagem tem motivado, desde a Antigüidade, intensos debates. Dentre as diferentes teorias propostas tem ganho relevância, nos séculos XX e XXI, a concepção cognitivista de David Ausubel, na qual se assume que os mecanismos do aprender são mais eficientes em situações nas quais o estudante consegue, utilizando conhecimentos prévios, agregar e incorporar significado aos novos conteúdos, evitando-se, assim, que esse conhecimento recém-incorporado seja armazenado, por meio de associações ineptas, à estrutura cognitiva. No âmbito da educação médica, diante das imperiosas mudanças necessárias para a formação de um profissional que aprenda a aprender, a aprendizagem significativa permite uma genuína contextualização dos conhecimentos necessários à prática médica, facultando, portanto, um aprendizado mais efetivo e permitindo a atuação em um contexto complexo e interdisciplinar. Em conformidade com o exposto, o presente manuscrito objetiva apresentar a teoria da aprendizagem significativa, discutindo elementos da incorporação deste conceito à educação médica.

\section{ABSTRACT}

The learning process has fosterd intense debate since ancient times - among the different proposed theories David Ausubel cognitivist conceptual frame of has found relevance during the $X X^{\text {th }}$ century through to the XXIrst. It assumes that learning mechanisms are more efficient in such situations when the student is able to aggregate and incorporate significance to new contents, by means of linking then to previous knowledge, thus avoiding that the knowledge be stored by way of inept associations in the cognitive structure. The scope of medical education demands the changes necessary for the formation of a professional able to know

Trabalho realizado nas cidades de Teresópolis e Nilópolis, Estado do Rio de Janeiro.

${ }^{1}$ Centro Universitário Serra Órgãos, Rio de Janeiro, Brasil

${ }^{2}$ Centro Federal de Educação Tecnológica de Química de Nilópolis, Rio de Janeiro, Brasil.

${ }^{3}$ Centro Universitário Serra Órgãos, Rio de Janeiro, Brasil; Centro Federal de Educação Tecnológica de Química de Nilópolis, Rio de Janeiro, Brasil 
how to learn; meaningful learning allows the genuine contextualization of knowledge needed by the medical practice, therefore permitting effective learning and practice in a complex interdisciplinary context. In conformity with the above exposed, the present article aims to present the theorie of significant learning, discussing elements of the integration of this concept into the medical education.

\section{INTRODUÇÃO}

A educação é um processo sistemático e interativo de troca de informações entre pessoas de uma dada comunidade, com o propósito específico de ajudá-las a elaborar conhecimentos e significados, incorporando-os a sua estrutura cognitiva e ao patrimônio cultural coletivo. ${ }^{1,2}$ Trata-se de uma atividade com grande repercussão na vida individual, mas que, por definição, diz respeito à tessitura da sociedade:

Antes de tudo, a educação não é uma propriedade individual, mas pertence por essência à comunidade. O caráter da comunidade imprime-se em cada um dos seus membros e é no homem, zwon politikou [animal político], muito mais que nos animais, fonte de toda a ação e de todo comportamento. Em nenhuma parte o influxo da comunidade nos seus membros tem maior força que no esforço constante de educar, em conformidade com o seu próprio sentir, cada nova geração. A estrutura de toda a sociedade assenta nas leis e nas normas escritas e não escritas que unem e unem os seus membros. Toda a educação é assim o resultado da consciência viva de uma norma que rege uma comunidade humana, quer se trate de uma família, de uma classe ou de uma profissão, quer se trate de um agregado mais vasto, como um grupo étnico ou um Estado. ${ }^{1}$

De fato, desde imemoráveis tempos, sabe-se que cada sistema de educação está claramente relacionado a estados sociais determinados, concretizando e justificando o ideal vista físico, intelectual e moral em que está inserida a sociedade. ${ }^{3}$ Logo, no atual contexto do mundo do trabalho em saúde, em função da crescente incorporação de novas tecnologias, há premência do surgimento de propostas para o desenvolvimento e reestruturação tanto do profissional quanto do processo de trabalho. ${ }^{4}$ Cabe, assim, às entidades formadoras atentar para o pensar e o utilizar de metodologias que permitam que o caminho do aprender se faça de modo mais harmônico, coerente e sustentável.

Os reflexos da tensa articulação entre sujeitos e coletividade e a percepção do que a sociedade demarca como ideal a ser atingido têm marcante influência no modo segundo o qual se compreende o processo ensino-aprendizagem, um dos aspectos significativos da educação. De fato, os modos pelos quais este se consubstancia tem sido mote de acérrimas polêmicas desde a Antigüidade, caracterizando-se correntes de inspiração racionalista e empirista, na dependência do elemento considerado preeminente: a razão/entendimento - como no caso da reminiscência platônica ${ }^{5,6}$ — ou a experiência/sensibilidade - como formulado pela idéia de mente lousa vazia, em John Locke. ${ }^{7,8}$

Estas vertentes foram habilmente compostas por Immanuel Kant em sua teoria do conhecimento, ${ }^{9}$ considerada "precursora" dos construtivismos, concepções que enfatizam o papel do sujeito cognoscente (sujeito epistêmico) na "construção" da realidade conhecida (objeto epistêmico). . Foi a partir de tais idéias que Jean Piaget compôs as bases do construtivismo. ${ }^{10} \mathrm{Em}$ linhas gerais, entende-se que a cognição se baseia em um mecanismo de processamento de informações, no qual os símbolos são utilizados como base em combinações. O conhecimento é, portanto, construído a partir da interação entre o sujeito e o objeto. ${ }^{11}$ No âmbito pedagógico, o professor passa a atuar como um problematizador, colocando o aprendiz em situações, nas quais a interação sujeito-objeto possa ocorrer, o que torna este último um sujeito ativo e participativo no seu processo de aprendizagem. É trazido à tona o conceito freireano da educação problematizadora, ou seja, aquela que representa o conjunto compartilhado entre dois sujeitos pensantes na busca de significados comuns. ${ }^{12}$

É propriamente neste horizonte - o construtivismo que se inscreve a teoria cognitivista de David Ausubel, a aprendizagem significativa. ${ }^{13}$ Para este autor, o movimento de aprender é mais eficiente nas ocasiões em que o estudante consegue agregar e incorporar, ao repertório de conceitos previamente organizados, os novos conteúdos, evitando, assim, que estes sejam armazenados, na estrutura cognitiva, por meio de associações espúrias. ${ }^{14}$ Com efeito,

Para haver aprendizagem significativa são necessárias duas condições. Em primeiro lugar, o aluno precisa ter uma disposição para aprender: se o indivíduo quiser memorizar o conteúdo arbitrária e literalmente, então a aprendizagem será mecânica. Em segundo, o conteúdo escolar a ser aprendido tem que ser potencialmente significativo, ou seja, ele tem que ser lógica e psicologicamente significativo: o significado lógico depende somente da natureza do conteúdo, e o significado psicológico é uma experiência que cada indivíduo tem. Cada aprendiz faz uma 
filtragem dos conteúdos que têm significado ou não para si próprio ${ }^{14}$ (p. 37-42).

Com base nestas premissas, compreender os referenciais teóricos da aprendizagem significativa — e colocá-los em prática - pode ser visto como elemento fundamental para os processos educativos em diferentes níveis, incluindo-se a formação científica e a formação de nível superior, tal como a exigida na graduação em Medicina, na qual a inter-relação de conteúdos e técnicas é aspecto primaz. Deste modo, no presente manuscrito propõe-se a reflexão, a partir de uma revisão conceitual dos principais aspectos da Teoria de David Ausubel, sobre a utilização da aprendizagem significativa no ensino médico.

\section{Ausubel e a aprendizagem significativa}

David Ausubel, judeu nascido, em 1918, na cidade de Nova York, graduou-se em Psicologia, tendo se destacado nas áreas de psicologia do desenvolvimento, psicologia educacional, psicopatologia e desenvolvimento do ego. ${ }^{15}$ Trabalhou durante toda a vida, aposentando-se apenas aos 75 anos (em 1994), ainda que se mantivesse produtivo pensando e escrevendo - , até sua morte.

A maior contribuição de Ausubel foi a proposição de uma teoria explicativa do processo de aprendizagem humana, embasada nos princípios organizacionais da cognição, valorizando, então, o conhecimento e o entendimento de informações e não meramente a memorização mecânica ("decoreba"). ${ }^{16}$ Tem como pressuposto principal a relação de conteúdos, que vão se agregando de forma hierarquizada e mais complexa de acordo com a ligação a conhecimentos prévios - os chamados subsunçores -, os quais funcionam como "âncoras", propiciando tanto a aprendizagem, quanto o crescimento cognitivo dos indivíduos. ${ }^{16,17,18}$

Desta forma, o processo de construção do conhecimento se dá de forma individualizada e correlacionada com a aprendizagem prévia, que o sujeito carrega em seu repertório cognitivo. ${ }^{19,20}$ Torna-se claro que a utilização das experiências trazidas por cada estudante é fundamental para que a ancoragem de conteúdos se dê de forma efetiva e duradoura, consistindo, assim, em aprendizagem significativa. ${ }^{15,17,18 ~ E ́}$ certo, contudo, que, em diversos momentos, torna-se necessária a abordagem de uma outra forma de aprendizagem, a mecânica — por exemplo, no treinamento de procedimentos e de rotinas, ainda que mesmo em tais circunstâncias permaneça a percepção de que a memorização simples, como aquela realizada no momento da aprendizagem mecânica, rapidamente leva à perda dos conhecimentos conquistados. ${ }^{15}$
A integração de novas informações, com a aprendizagem significativa, facilita a aplicação do conhecimento em atividades mais complexas, como, por exemplo, na apreciação de casos clínicos, importantes para os estudos na área de saúde. ${ }^{20} \mathrm{~A}$ aprendizagem significativa deve incentivar o estudante a aplicar a informação de forma prática; ela, assim, integra-se mais facilmente - e de forma mais completa - , sendo valorizada de acordo com seu significado.

Ausubel propõe que a rede de conhecimento se construa através da associação da nova informação, a qual está sendo vista pela primeira vez, a conhecimentos já aprendidos e vivenciados, isto é, a nova informação deve ser incluída em um cabedal de conhecimentos prévios. Após essa relação, consolidada pela agregação aos "subsunçores", cria-se um novo e/ou mais abrangente conceito. Ao se somar, nesse processo, a experimentação ao estímulo e interação com o aprendiz, tem-se maior chance de obtenção de uma aprendizagem realmente significativa. ${ }^{15,17,18,20}$ Neste ponto, vale ressaltar que o equilíbrio cognitivo é certamente um estado dinâmico, sendo capaz de construir e manter a ordem funcional e estrutural do sistema ${ }^{10}$ num eterno processo de construção-desconstrução-reconstrução.

Aprender significativamente é, pois, o grande objetivo. Os padrões dogmáticos de uma educação disciplinadora e bancária ${ }^{21}$ são quebrados e a relação professor/aluno se transforma em uma relação mestre/aprendiz, sem a rigidez de papéis pré-fixados. Há, então, flexibilização da participação dos atores, na medida em que o aprendiz é também mestre e vice-versa. ${ }^{15}$ Ademais, o professor se comporta como modelo para os alunos, ao incentivá-los ao exercício da aprendizagem significativa, $^{20}$ tendo sempre em mente que o ser humano é um sujeito inacabado - logo, em constante elaboração. ${ }^{22}$

Nesses processos, a interação entre o novo conhecimento e o já existente faz com que ambos se transformem. ${ }^{16} \mathrm{O}$ conhecimento adquirido terá mais consistência, já que será modificado, integrado e usado, não apenas no momento da aprendizagem, mas em futuras situações de ensino e de vida. ${ }^{20}$ Pode-se concluir que tal aprendizagem, idiossincrática e particular, se relaciona com a estrutura de cada indivíduo e, que, ao mesmo tempo, exerce o poder de mudança sobre o indivíduo, assim como sofre o poder de ser mudada. ${ }^{15,16}$

Os meios usados na construção desse conhecimento são variados, merecendo destaque (1) a diferenciação progressiva onde os conceitos que interagem com os novos conhecimentos, e servem de base para atribuição de novos significados, vão se modificando em função desta interação - e (2) a reconciliação integrativa - que consiste no estabelecimento de relações entre idéias, conceitos e proposições já existentes na estrutura 
cognitiva. Esses processos têm como resultado explícito o delineamento de diferenças e similaridades entre as idéias relacionadas. ${ }^{16}$

Os princípios da aprendizagem significativa trazem inúmeras possibilidades de aplicação em diferentes áreas, ${ }^{23,24}$ incluída a formação profissional em saúde - p. ex., na educação médica - colaborando para um ganho cognitivo real do estudante e, de fato, para o alcance do verdadeiro ideário do professor/mestre: contribuir para a formação do homem. ${ }^{1}$

\section{A aprendizagem significativa e a educação médica}

O movimento de mudança existente na educação médica não é uma novidade no Brasil ${ }^{25} \mathrm{e}$, certamente, tem como bases diversas características do mundo atual, tais como (1) a globalização, (2) a rapidez na produção e na circulação das informações, (3) as diferentes necessidades de saúde da população - levando-se em consideração que se trata de um país continental, com diferentes perfis culturais, econômicos e sociais -, (4) a crescente incorporação de tecnologia à medicina e (5) a necessidade de formação de um profissional crítico e reflexivo, capaz de atuar em equipe multidisciplinar em diversos cenários da prática. ${ }^{26,27,28,29,30} \mathrm{~A}$ imposição da transformação diz respeito à constatação da insuficiência das instituições e dos currículos tradicionais - subdivididos em áreas básica e profissional e fragmentados em disciplinas para a formação ambicionada. Neste sentido, as metodologias ativas têm se constituído em uma salutar alternativa, ao permitir a promoção da desejada articulação entre a universidade, o serviço - sobretudo, o Sistema Único de Saúde (SUS) - e a comunidade, ${ }^{31}$ assim como o ajuste do processo de ensinagem na formação do médico, foco imprescindível para que a abrangência das necessidades seja alcançada. Para isso, deve haver um olhar sobre a nova prática de formação, que inclua transformações na concepção, no planejamento e na construção de conteúdos e objetivos educacionais, os quais devem ser revistos e transformados:

- De orientada por conteúdos e objetivos mal definidos, para orientada para competências bem-definidas e baseadas nas necessidades de saúde das pessoas;
- Da transmissão de informações e pura utilização da memória (decorar), para a construção do conhecimento $e$ desenvolvimento de habilidades e atitudes para resolver problemas, considerando experiências anteriores de aprendizagem, culturais e de vida;

- De professores capacitados somente em conteúdos para professores capacitados também em educação médica;

- Do ensinar-aprender com observação passiva dos estudantes, para ao aprender a aprender, com participação ativa dos aprendizes, ou seja, do centrado no professor para centrado no estudante;

— Da humilhação e intimidação dos estudantes pelos docentes, para o respeito mútuo;

- Da clássica inquirição do professor 'como você ainda não sabe?', para 'o que você ainda não sabe?', ajudando o estudante a identificar e superar hiatos de aprendizagem;

- De primeiro a teoria e depois a prática (somente nos últimos anos do curso), para um processo integrado de ação-reflexãoação, desde o princípio do curso;

- De uma atenção episódica, centrada na doença, para uma atenção contínua, centrada no cuidado das pessoas, com o estabelecimento de vínculos afetivos com elas;

- Da utilização do paciente puramente como objeto de prática dos estudantes, para a participação consentida e informada do paciente no processo de ensino-aprendizagem com respeito a sua dignidade e privacidade;

- Do uso de campos de prática predominantemente hospitalares, para cenários de ensino-aprendizagemassistência onde os estudantes sejam inseridos como membros ativos;

- De avaliação praticamente somativa/punitiva no final das unidades, estágios e disciplinas, para uma avaliação preferencialmente formativa, com constante feedback ${ }^{32}$ (p. 40-61).

As metodologias ativas trazem como características maiores, (1) servir para o fortalecimento da autonomia do estudante $^{22}$ e (2) fomentar a indissociabilidade teoria-prática, ${ }^{33}$ estribando-se, conceitualmente, na aprendizagem 
significativa, ${ }^{13}$ consistente referencial teórico para a construção de um saudável processo de ensinar-aprender.

A aprendizagem significativa não é somente utilizável, mas essencial como instrumento para que se dê o ganho cognitivo buscado em um contexto onde o estudante é agente ativo da construção de seu saber. Logo, é pungente a necessidade de professores atuantes nas diversas áreas do conhecimento - essenciais na constituição do saber-fazer médico - mergulharem nas águas da pedagogia, importante auxílio para a constituição da complexa rede de conhecimentos, habilidades psicomotoras e demais competências a serem adquiridas pelo profissional em sua fase de formação.

\section{Aprendizagem significativa e práxis: os mapas conceituais}

Com o intuito de ajudar no curso da aprendizagem significativa, Moreira (1988) ${ }^{16}$ propõe a utilização de mapas conceituais, instrumentos que favorecem que o professor identifique na estrutura cognitiva de seus alunos, os subsunçores, organizadores essenciais para os conceitos a serem estudados sobre um determinado assunto. Concebidos por Novak (1984), tais mapas representariam a concretização da aprendizagem significativa, auxiliando no delineamento das estruturas cognitivas. ${ }^{16}$ De fato, as mudanças provocadas na estrutura cognitiva dos alunos com o uso de mapas conceituais são evidentes quando se observam os estudos sobre seu rendimento. Na investigação realizada por Rendas e colaboradores, ${ }^{34}$ em ensino de saúde (curso de fisiopatologia), foi demonstrada a contribuição, inclusive, para a eliminação de pontos fracos e inconsistências. É visível, também, o crescimento da complexidade na elaboração dos mapas com a adição de mais tópicos e criação de maiores inter-relações, com o aprofundamento da questão apreendida. ${ }^{16,18}$

Um outro aspecto da utilização de mapas conceituais refere-se ao favorecimento da atividade docente no momento de distinguir os diferentes tipos de conteúdo (curricular e instrumental) - permitindo que o mesmo focalize sua atenção, e a dos alunos, naqueles conhecimentos que devem ser apreendidos —, além do auxílio na identificação dos conhecimentos que propiciam a obtenção desse novo aprendizado. Ou seja, todos os participantes atuarão ativamente sobre a construção desse novo conhecimento e de seus significados. Neste aspecto, Moreira (1988) afirma que:

Mapas conceituais foram desenvolvidos para promover a aprendizagem significativa. A análise do currículo e o ensino sob uma abordagem ausubeliana, em termos de significados, implicam: 1) identificar a estrutura de significados aceita no contexto da matéria de ensino; 2) identificar os subsunçores (significados) necessários para a aprendizagem significativa da matéria de ensino; 3) identificar os significados preexistentes na estrutura cognitiva do aprendiz; 4) organizar seqüencialmente o conteúdo e selecionar materiais curriculares, usando as idéias de diferenciação progressiva e reconciliação integrativa como princípios programáticos; 5) ensinar usando organizadores prévios, para fazer pontes entre os significados que o aluno já tem e os que ele precisaria ter para aprender significativamente a matéria de ensino, bem como para o estabelecimento de relações explícitas entre o novo conhecimento e aquele já existente e adequado para dar significados aos novos materiais de aprendizagem ${ }^{16}$ (p. 87-95).

Em consonância com tais formulações, compreende-se que os mapas conceituais se adequam bem ao ensino de ciências da saúde, ao permitirem, tanto ao professor quanto ao aluno, identificar a "hierarquia" e promover a contextualização dos conhecimentos. Isso só se torna possível por ser uma técnica flexível e de fácil aplicação, podendo ser utilizada em diferentes etapas da aprendizagem, com finalidades diversas. ${ }^{35}$ Entretanto, ainda que a validade dos mapas conceituais seja amplamente reconhecida - tanto na melhoria da capacidade de articulação das idéias, quanto no desenvolvimento do trabalho em grupo - , estes não devem ser considerados como instrumentos únicos do ensino - ou ainda, como metodologia exclusiva da aprendizagem significativa - cabendo, sempre, a (re)discussão sobre os diferentes modos de se tornar significativo o que se aprende.

\section{Ponderações derradeiras}

A aprendizagem significativa tem se mostrado uma corrente pedagógica importante, devendo ser considerada no processo de educação em diversas áreas do saber — o olhar, neste texto, dirigiu-se à formação médica —, na medida em que permite, a docentes e discentes, a revisão e a (re)pactuação da relação existente entre ambos. Tal perspectiva favorece uma participação mais ativa do indivíduo em construção, sendo o conhecimento ancorado de forma consistente, sendo especialmente útil, neste sentido, o emprego dos mapas conceituais, os quais favorecem a associação e a inter-relação entre os antigos e os novos conceitos.

Ao se considerar a graduação médica como uma singular viagem, realizada pelo aprendiz, pelas correntezas do saberfazer próprio da medicina, é mister que se utilizem âncoras peças de ferro forjado destinada a reter o navio no ponto em que se $q_{u e r}^{36} \mathrm{e}$, por extensão de sentido o que serve para proteger, para amparar; arrimo, abrigo, esteio, amparo ${ }^{36}$ — e mapas — representações 
gráficas e convencionais, em papel, cartolina, tela etc., dos dados referentes à superfície do globo terrestre, a uma região dessa superfície, à esfera celeste; carta geográfica ${ }^{36}$ — instrumentos que podem auxiliar, protegendo e guiando, no percurso em direção à formação de um médico mais apto a responder às atuais demandas da sociedade. Tal é o genuíno sentido de uma infrene busca da arca perdida.

\section{REVISADO PELO AUTOR}

\section{REFERÊNCIAS}

1. Jaeger W. Paidéia: a formação do homem grego. São Paulo: Martins Fontes; 1995.

2. Mcgriff SJA. Position paper and concept map: philosophy of education, 2000. Disponível em: www.personal.psu.edu/ sjm256/. Acessado em 10 de dezembro de 2006.

3. Hubert R. História da pedagogia. São Paulo: Companhia Editora Nacional; 1976.

4. Martins MIC. A transição tecnológica na saúde: desafios para a gestão do trabalho. Trabalho, Educação e Saúde 2004; 2(2): 287-310.

5. Platão. Mênon. Rio de Janeiro: Editora da PUC-Rio / Edições Loyola; 2001.

6. Siqueira-Batista R, Schramm FR. Platão e a medicina. História, Ciências, Saúde - Manguinhos 2004; 11(3): 619-634.

7. Hessen J. Teoria do conhecimento. São Paulo: Martins Fontes; 2000.

8. Locke J. Ensaio acerca do entendimento humano. São Paulo: Nova cultural; 2000.

9. Kant I. Crítica da razão pura. Lisboa: Calouste Gulbenkian; 1994.

10. Piaget J. A equilibração das estruturas cognitivas. Problema Central do Desenvolvimento. Rio de Janeiro: Zahar; 1976.

11. Piaget J. Seis estudos de psicologia. $24^{\mathrm{a}}$ ad. Rio de Janeiro: Forense; 2003.

12. Vasconcelos MLMC, Brito, RH. Conceitos de educação em Paulo Freire. Petrópolis, Rio de Janeiro: Vozes; São Paulo: Mack Pesquisa; 2006.

13. Ausubel DP. A aprendizagem significativa: A teoria de David Ausubel. SP: Moraes; 1982.

14. Pelizzari A, Kriegl ML, Baron MP, Finck NTL, Dorocinski SI. Teoria da aprendizagem significativa segundo Ausubel. Rev PEC. 2001- 2002; 2(1): 37-42.

15. Ausubel DP. Ausubel. www.davidausubel.org; acessado em 04/12/2006.
16. Moreira MA. Mapas conceituais e aprendizagem significativa. (Texto Adaptado e atualizado, em 1997, de um trabalho com o mesmo título publicado em $\mathrm{O}$ ENSINO, Revista Galaico Portuguesa de Sócio-pedagogia e sócio-linguística, Pontevedra 1988; 23 (28): 87-95.

17. Hassad J. Backup of meaningful learning model. Dear Habermas Current Issue 2003; 17 (3).

18. Kearsley G. Subsumtion theory (D. Ausubel), 2006. Disponível em: http: / / tip.psychology.org/ausubel.html. Acessado em 11 de outubro de 2006.

19. Buchweitz B. Aprendizagem significativa: idéias de estudantes concluintes do ensino superior. Investigações em Ensino de Ciências 2001; 6(2). Disponível em: http:/ / www.if.ufrgs.br/public/ensino/vol6/n2/ v6_n2_a2.htm. Acessado em 02 de abril de 2006.

20. Michael J. In pursuit of meaningful learning. Advances in Physiology Education 2001; 25(3): 145-158.

21. Freire P. Pedagogia do oprimido. $38^{\mathrm{a}}$ ed. São Paulo: Paz e Terra; 2004.

22. Freire P. Pedagogia da autonomia. $33^{\underline{a}}$ ed. São Paulo: Paz e Terra; 2006.

23. Nardin CS, Salgado TDM, Del Pino JC. Análise de uma proposta de ensino de reações químicas entre compostos inorgânicos referenciada em mecanismos de reação. In: V Encontro Nacional de Pesquisa em Educação, 2005.

24. Oliveira RA, Cyrino MCCT. A compreensão de duas professoras de matemática sobre o modo como seus alunos aprendem. In: III Seminário Internacional de Pesquisa em Educação Matemática, 2006, Águas de Lindóia. Anais do III Seminário Internacional de Pesquisa em Educação Matemática 2006.

25. Pontes AC, Rego S, Silva Junior AG. Saber e prática docente na transformação do ensino médico. Rev Bras Edu Méd 2006; 30(2): 66-75.

26. Behrens MA. O paradigma emergente e a prática pedagógica. Petrópolis, RJ: Vozes; 2005.

27. Brasil. Lei n.9.394 de 20 de dezembro de 1996. Estabelece as diretrizes da Educação Nacional - DOU; 23 de dezembro de 1996; Brasília (DF); p. 833-41.

28. Brasil. Conselho Nacional de Educação, Câmara de Educação Superior. Diretrizes Curriculares Nacionais do Curso de Graduação em Medicina. Resolução CNE / CES №. 4, 7 de novembro de 2001; Brasília (DF); p.1-6.

29. Da Ros MA. A ideologia nos cursos de medicina. In: Marins JJN, Rego S, Lampert JB, Araújo JGC. In: Educação médica em transformação: instrumentos para 
a construção de novas realidades. São Paulo: Hucitec; Rio de Janeiro: Associação Brasileira de Educação Médica; 2004. p. 224-44.

30. Oliveira GS, Koifman L. Integralidade do currículo de medicina: inovar/transformar, um desafio para o processo de formação. In: Marins JJN, RegoS, Lampert JB, AraújoJGC. Educação médica em transformação: instrumentos para a construção de novas realidades. São Paulo: Hucitec; Rio de Janeiro: Associação Brasileira de Educação Médica; 2004. p.143-64.

31. Feurwerker LCM, Sena RR. A contribuição ao movimento de mudança na formação profissional em saúde: uma avaliação das experiências UNI. Interface - Comunicação, Saúde e Educação 2002; 6(10): 37-50.

32. Zanolli M. Metodologias ativas de ensino-aprendizagem na área clínica. In: Marins JJN, RegoS, LampertJB, Araújo JGC. Educação médica em transformação: instrumentos para a construção de novas realidades. São Paulo: Hucitec; Rio de Janeiro: Associação Brasileira de Educação Médica; 2004. p. 40-61.

33. Venturelli J. Educación Médica. Novos enfoques, metas y métodos. 2a Ed. Organización Panamericana de la Salud, Washington; 2003.
34. Rendas AB, Fonseca M, Pinto PR. Toward meaningful learning in undergraduate medical education using concept maps in a PBL pathophysiology course. Advances in Physiology Education 2006; 30: 23-29,

35. Moreira MA, Buchweitz B. Novas estratégias de ensino e aprendizagem: os mapas conceituais e o vê epistemológico. Lisboa: Plátano Edições Técnicas; 1993.

36. Houaiss A. Dicionário Houaiss da língua portuguesa . Rio de Janeiro: Objetiva; 2001.

\section{CONFLITOS DE INTERESSE}

Declarou não haver.

\section{ENDEREÇO PARA CORRESPONDÊNCIA}

Andréia Patrícia Gomes

Centro Universitário Serra dos Orgãos - UNIFESO

Av. Alberto Torres, 111 - Alto

Teresópolis - RJ

Cep.: 25964-000 